\title{
Optimized octave-spanning Ti:sapphire laser oscillator characterized by novel two-dimensional shearing interferometry
}

\author{
Shih-Hsuan Chia ${ }^{1,2}$, Fabian Scheiba ${ }^{1,2}$, Giulio M. Rossit ${ }^{1,2}$, Oliver D. Mücke ${ }^{1,2}$, and Franz X. Kärtner ${ }^{1,2}$ \\ ${ }^{1}$ Center for Free-Electron Laser Science, Deutsches Elektronen-Synchrotron DESY, Notkestraße 85, 22607 Hamburg, Germany \\ ${ }^{2}$ Physics Dept., University of Hamburg and the Hamburg Center for Ultrafast Imaging, Luruper Chaussee 149, 22761 Hamburg, Germany
}

Stable ultra-broadband pulse generation via Kerr-lens mode locking (KLM) paves the way for scientific explorations in many fields, such as optical metrology, study of ultrafast dynamics, and nonlinear light microscopy. For example, Ti:sapphire laser oscillators, capable of generating octave-spanning spectra with potentially close-to-single-cycle waveforms, have been employed as optical flywheels providing a very precise timing reference [1], and to study waveform-sensitive interactions with solid-state nanostructures [2]. Despite the experimental demonstrations of octave-spanning spectra from Ti:sapphire oscillators, the intracavity spatiotemporal pulse dynamics has not been completely understood and optimized, and the pulse characterization still remains challenging. Although the use of double-chirped mirror (DCM) pairs provide octave-spanning dispersion compensation, the residual dispersion oscillations, originating from the chirped mirrors, play adverse roles in few-cycle KLM dynamics. Residual intracavity phase oscillations tend to generate satellite pulses, which hamper the onset of stable mode locking. Moreover, the interplay between such residual phase oscillations and optical Kerr nonlinearity limits the output spectral bandwidth, as well as leading to strong modulations in both longitudinal and spatial modes. Therefore, the improvements of the output spectrum, beam quality, and mode-locking stability require a more precise intracavity dispersion control. A phase-optimized cavity has been designed and fabricated delivering $<0.1$ rad of intracavity residual phase over the whole resonating bandwidth of $0.65 \mu \mathrm{m}-1.14 \mu \mathrm{m}$. Fig. 1(a) shows the experimental spectrum (blue), in excellent agreement with the numerical simulation (grey), from the optimized cavities with $5 \%$ output coupling, as well as the previous state-of-the-art output, having a 3.6-fs transform-limited (TL) pulse duration (green). $>10$-dB enhancements in power spectral density around both the center gain region and $1140 \mathrm{~nm}$ are obtained, suggesting the improvements in terms of both power and precision locking control to be beneficial for the use as optical frequency comb. In the time domain (see the inset of Fig. 1(a)), a compressed pulse with 4.3-fs duration (3.9-fs TL from the $5 \%$ spectrum) is characterized by a newly designed two-dimensional shearing interferometry (2DSI) scheme. Instead of chirping two replicas of the initial pulse [3], the narrowband ancillary pulses are generated by means of inteference filters inside a Michelson interferometer featuring a piezo-controlled arm length, allowing to generate the 2DSI fringe pattern shown in the inset of Fig. 1(b). The filtering approach, avoiding stretchingratio calculations, gives a precise control over the shear frequency that plays a key role in the reconstruction of the electric field. In addition, the simplicity in choosing the wavelengths of the ancillae just by replacing the filters makes our 2DSI setup flexible to characterize pulses with different parameters. A better result toward single-optical-cycle pulses is expected for optimized cavities using $1 \%$ output coupling. The compressed pulses will be used to study field-controlled, carrier-envelope phase-sensitive photoemission from arrays of tailored metallic nanoparticles [2]. We will provide experimental results and detailed discussions in the presentation.
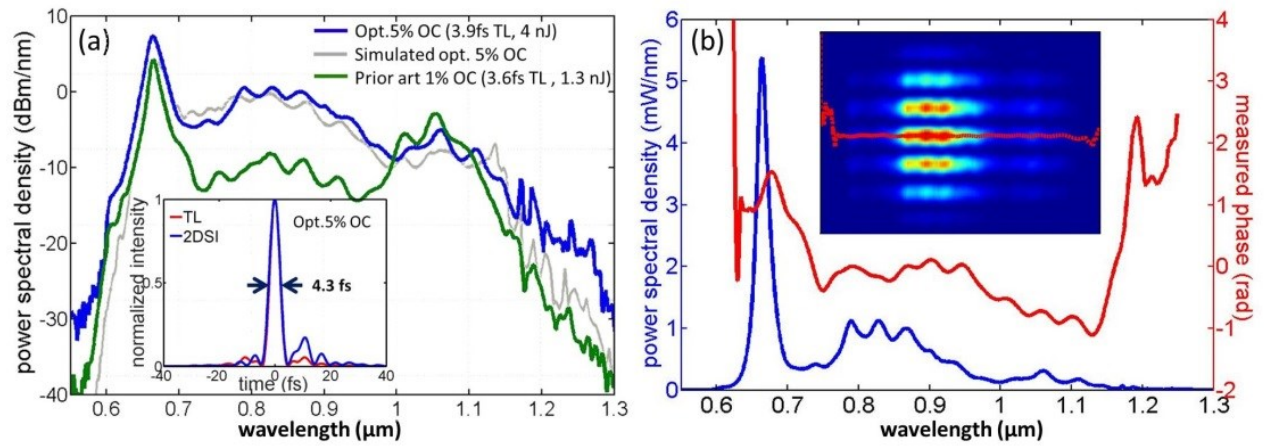

Fig. 1. (a) The experimental spectrum (blue), in excellent agreement with the numerical simulation (grey), from the optimized cavities with $5 \%$ output coupling, as well as the previous state-of-the-art output with a 3.6 transform-limited (TL) pulse duration (green). The inset shows the pulse (blue) retrieved using the novel 2DSI setup, 4.3fs, compared with the TL one (red), 3.9 fs. (b) The experimental spectrum (blue) with optimized 5\% output coupling and the measured phase (red). The acquired 2DSI traces are shown in the inset as well as the retrieved group delay (red).

\section{References}

[1] A. J. Benedick, J. G. Fujimoto, and F. X. Kärtner, "Optical flywheels with attosecond jitter, " Nat. Photonics 6, 97-100 (2012).

[2] W. P. Putnam, R. G. Hobbs, P. D. Keathley, K. K. Berggren, and F. X. Kärtner, "Optical-field-controlled photoemission from plasmonic nanoparticles," Nat. Physics AOP, 3978 (2016).

[3] J. R. Birge, R. Ell, and F. X. Kärtner "Two-Dimensional Spectral Shearing Interferometry (2DSI) for Ultrashort Pulse Characterization" Opt. Lett. 31, 2063-2065 (2006). 